\title{
Identification of potential key genes and pathways in hepatitis B virus-associated hepatocellular carcinoma by bioinformatics analyses
}

\author{
XIANG ZHANG $^{1 *}$, LINGCHEN WANG ${ }^{2,3 *}$ and YEHONG YAN ${ }^{1}$ \\ ${ }^{1}$ Department of General Surgery, The First Affiliated Hospital of Nanchang University; \\ ${ }^{2}$ Department of Biostatistics and Epidemiology, School of Public Health; \\ ${ }^{3}$ Jiangxi Provincial Key Laboratory of Preventive Medicine, \\ Nanchang University, Nanchang, Jiangxi 330006, P.R. China
}

Received July 16, 2019; Accepted January 24, 2020

DOI: $10.3892 / \mathrm{ol} .2020 .11470$

\begin{abstract}
Chronic hepatitis B virus (HBV) is one of the leading causes of hepatocellular carcinoma (HCC). The precise molecular mechanisms by which HBV contributes to HCC development are not fully understood. The key genes and pathways involved in the transformation of nontumor hepatic tissues into $\mathrm{HCC}$ tissues in patients with $\mathrm{HBV}$ infection are essential to guide the treatment of HBV-associated HCC. Five datasets were collected from the Gene Expression Omnibus database to form a large cohort. Differentially expressed genes (DEGs) were identified between HCC tissues and nontumor hepatic tissues from HBV-infected patients using the 'limma' package. The top 50 upregulated and top 50 downregulated DEGs in HCC vs. nontumor tissues were demonstrated in subsets by heat maps. Based on the DEGs, Gene Ontology functional and Kyoto Encyclopedia of Genes and Genomes pathways enrichment analyses were performed. Several key pathways of the up- and downregulated DEGs were identified and presented by protein-protein interaction (PPI) networks. A total of 1,934 DEGs were identified. The upregulated DEGs were primarily associated with the 'cell cycle'. Among the DEGs enriched in the 'cell cycle' pathway, 6 genes had a $\log _{2}$-fold change $>2$ : SFN, BUB1B, TTK, CCNB1, CDK1 and $C D C 20$. The downregulated DEGs were primarily associated with the metabolic pathways, such as 'carbon metabolism', 'glycine, serine and threonine metabolism', 'tryptophan
\end{abstract}

Correspondence to: Dr Yehong Yan, Department of General Surgery, The First Affiliated Hospital of Nanchang University, 17 Yong Wai Zheng Street, Nanchang, Jiangxi 330006, P.R. China E-mail: yyh711@126.com

*Contributed equally

Key words: differentially expressed genes, hepatocellular carcinoma, hepatitis B virus, signaling pathway, cell cycle, metabolic pathways metabolism', 'retinol metabolism' and 'alanine, aspartate and glutamate metabolism'. The DEGs in the 'cell cycle' and 'metabolic pathways' were presented by the PPI networks respectively. Overall, the present study provides new insights into the specific etiology of $\mathrm{HCC}$ and molecular mechanisms for the transformation of nontumor hepatic tissues into HCC tissues in patients with a history of HBV infection and several potential therapeutic targets for targeted therapy in these patients.

\section{Introduction}

Hepatocellular carcinoma (HCC) is a prevalent malignant liver disease (1). In most cases, viral infection contributes to the development, invasion and metastasis of HCC (2), which is a global public health problem. In particular, chronic hepatitis B virus (HBV) is one of the leading causes of HCC with an estimated 400 million individuals currently affected by chronic infection worldwide $(3,4)$. More than $50 \%$ of HCC cases arise from chronic HBV infections $(5,6)$. In high-prevalence areas, chronic $\mathrm{HBV}$ infection is estimated to account for over $80 \%$ of HCC cases (7). Moreover, patients with HBV-associated HCC have notably higher rates of metastasis and recurrence compared with those without HBV infection $(8,9)$. Three-quarters of the world's population live in areas where there are high levels of HBV infection (10). However, the currently available antiviral agents can barely eliminate chronic HBV infection (11). HBV-associated liver diseases cause approximately 1 million deaths per year (3), driving an intensive search for curative treatment approaches (12). Han et al (13) reported that WNT family gene expression is associated with the development of HBV-associated HCC. Tian and Ou's study (14) found that chronic HBV infection could lead to chronic inflammation in the liver, which could cause normal liver cells to transform into cancer cells (15). Although the correlation between chronic HBV infection and HCC development is strong, the precise molecular mechanisms by which HBV contributes to HCC development are not fully understood (16). Therefore, a clearer understanding of the molecular mechanisms of the transformation of nontumor hepatic tissues into HCC tissues 
in patients with HBV infection is required to guide the treatment of HBV-associated HCC (17).

A large array of data could be analyzed, given the remarkable development of high-throughput technologies for the profiling of genome-wide methylation and expression, such as methylation microarray and MeDip-seq, and RNA-seq, and the datasets publicly available worldwide (18). Potential biomarkers and signaling pathway associated with tumor regression could be identified using bioinformatics methods.

Thus far, there are insufficient bioinformatics studies focusing on the differentially expressed genes (DEGs) between HCC tissues and nontumor tissues from HBV-infected patients based on a large sample size. In the present study, data from more datasets on the same platform were collected in order to increase the sample size. Using a large cohort, DEGs between HCC tissues and nontumor tissues were identified. Furthermore, Gene Ontology (GO) functional enrichment analysis and Kyoto Encyclopedia of Genes and Genomes (KEGG) pathway enrichment analysis of the DEGs were performed. In addition, protein-protein interaction (PPI) networks were constructed based on the most enriched pathways. The results of the present study may help to identify key biomarkers for the personalized treatment of patients with HCC and a history of HBV infection, and provide further insights into tumor progression and further studies on HCC.

\section{Materials and methods}

Microarray datasets for differential expression analyses. A comprehensive search was conducted for HCC microarray datasets, including tissue samples from $\mathrm{HBV}$-infected patients in the Gene Expression Omnibus (GEO) database of the National Center for Biotechnology Information (http://www. ncbi.nlm.nih.gov/geo/) website. All the data of the selected datasets [GSE17548 (19), GSE55092 (20), GSE62232 (21), GSE84044 (22) and GSE84402 (23)] were produced from the GPL570 platform. Subsequently, the raw intensity files (CEL) of the datasets were downloaded from the GEO database. The robust multiarray average method of the R package 'affy' (version 1.60.0; https://bioconductor.org/packages/affy/) was used to process the raw intensity files and generate a large gene expression matrix of all the selected samples from the datasets meeting the criteria (HBV positive liver tissue samples with status information) for differential expression analyses (24). The matrices for each selected dataset were also generated. In addition, we used two independent HCC datasets, The Cancer Genome Atlas (TCGA; https://portal.gdc.cancer. gov/projects/TCGA-LIHC) and GSE76427 (25), including $\mathrm{HCC}$ patients without $\mathrm{HBV}$ infection to further elucidate the specificity of the expression of target genes in $\mathrm{HBV}$-associated HCC.

Analyses of DEGs. Within the large cohort, the DEGs between HCC tissues and nontumor hepatic tissues were identified using the R package 'limma' (version 3.38.3; https://bioconductor.org/packages/limma/), which is based on unpaired t-test (26); with the thresholds of $\log _{2}$-fold change $>1$ or $<-1$ and adjusted P-value $<0.05$. The results of the differential expression analyses were visualized with a volcano plot using the R package 'ggplot2' (version 3.1.0; https://bioconductor. org/packages/ggplot2/). The top 50 upregulated and top 50 downregulated DEGs were represented by heatmaps using the $\mathrm{MeV}$ software (version 4.9.0; http://mev.tm4.org/) in the selected datasets. The unsupervised hierarchical clustering of the selected genes and samples in the heatmaps was performed using an average linkage method using Pearson's correlation.

Enrichment analysis of $G O$ function and KEGG pathway. GO (http://www.geneontology.org) function and KEGG (https://www.kegg.jp/) pathways enrichment analyses of the upregulated and downregulated DEGs were performed using the WEB-based GEne SeT AnaLysis Toolkit (http://www. webgestalt.org/) via a significance threshold of false discovery rate $(\mathrm{FDR})<0.05$, in order to understand the critical biological implications of the identified DEGs in HBV-positive HCC tissues.

PPI network analyses. To further understand the direct and indirect associations among the DEGs, PPI networks of the upregulated and downregulated DEGs based on the top pathways of the KEGG pathway enrichment analysis were constructed and visualized using the Search Tool for the Retrieval of Interacting Genes/Proteins (https://string-db.org) database. The aforementioned methods are summarized in Fig. 1.

\section{Results}

Selection of microarray datasets for differential expression analysis. From the GEO database of NCBI, five datasets (GSE17548, GSE55092, GSE62232, GSE84044 and GSE84402) that met the study criteria were used for differential expression analyses. Within the five datasets, $321 \mathrm{HBV}$-positive samples with valid hepatic tissue status were selected to generate the gene expression matrix; 82 of which were tumor tissues, and 239 of which were nontumor tissues (Table I). The clinical characteristics of enrolled subjects can be found in supplementary data (Table SI).

DEGs in HCC tissues compared with nontumor hepatic tissues. The expression values of 42,901 genes among 82 HCC samples were compared with 239 nontumor hepatic samples from five GEO datasets. A total of 1934 DEGs were identified with the thresholds of fold change $>1$ or $<-1$ and adjusted P-value $<0.05$, including 682 upregulated genes and 1,252 downregulated genes. All DEGs are marked as red dots in the volcano plot (Fig. 2). In addition, the top 50 upregulated genes and downregulated genes are listed in Tables II and III, respectively (the top 100 up- and downregulated genes are listed in Tables SII and SIII, respectively). The heat maps of GSE17548, GSE55092 and GSE84402 demonstrate the expression of the top 100 DEGs (50 upregulated and 50 downregulated) in different subsets (GSE17548 and GSE84402 in Fig. 3; GSE55092 in Fig. S1).

GO functional and KEGG pathway enrichment analyses of the upregulated and downregulated DEGs. In order to understand the biological implications of the identified DEGs 
Table I. Characteristics of the datasets used in this study.

\begin{tabular}{lrrll}
\hline & \multicolumn{2}{c}{ Samples (n) } & & \\
\cline { 2 - 4 } Dataset & HCC & Non-tumor & Tissue & Platform \\
\hline GSE17548 & 10 & 11 & HBV hepatic tissue & Affymetrix Human Genome U133 Plus 2.0 Array \\
GSE55092 & 49 & 91 & HBV hepatic tissue & Affymetrix Human Genome U133 Plus 2.0 Array \\
GSE62232 & 10 & 0 & HBV hepatic tissue & Affymetrix Human Genome U133 Plus 2.0 Array \\
GSE84044 & 0 & 124 & HBV hepatic tissue & Affymetrix Human Genome U133 Plus 2.0 Array \\
GSE84402 & 13 & 13 & HBV hepatic tissue & Affymetrix Human Genome U133 Plus 2.0 Array
\end{tabular}

HCC, human hepatocellular carcinoma; HBV, hepatitis B virus.

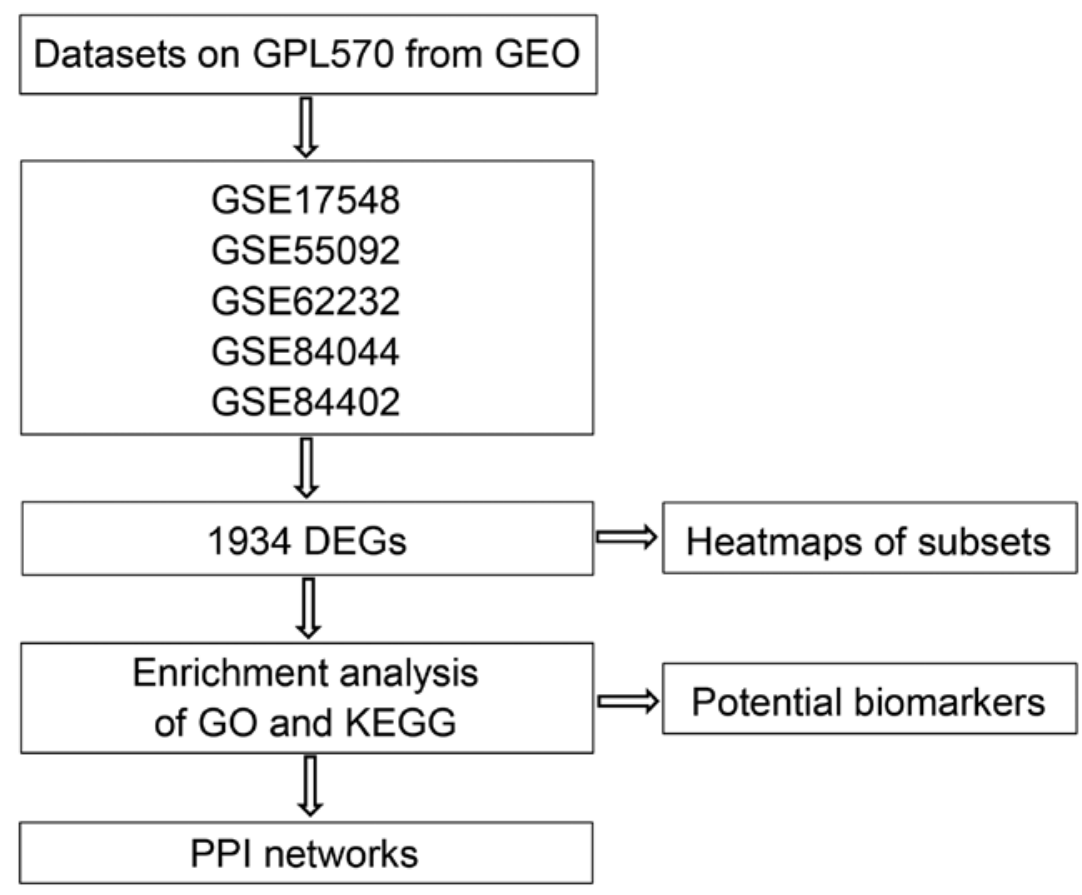

Figure 1. The process of identifying key genes and pathways in hepatitis B virus-associated hepatocellular carcinoma. GEO, Gene Expression Omnibus; DEGs, differentially expressed genes; GO, Gene Ontology; KEGG, Kyoto Encyclopedia of Genes and Genomes; PPI, protein-protein interaction.

in HBV-positive HCC tissues, GO functional and KEGG pathway enrichment analyses of the identified DEGs were performed. The GO terms of the up- and downregulated DEGs are presented in Fig. 4.

In the GO biological process category, upregulated DEGs were closely associated with the 'biological regulation' and 'metabolic process' terms, whereas the downregulated DEGs were closely associated with the 'metabolic process' and 'biological regulation' terms.

In the GO cellular component category, upregulated DEGs were closely associated with the 'nucleus' and 'membrane' terms, whereas the downregulated DEGs were closely associated with the 'membrane' and 'vesicle' terms.

In the GO molecular category, upregulated DEGs were closely associated with the 'protein binding' and 'nucleic acid binding' terms, whereas the downregulated DEGs were closely associated with the 'protein binding' and 'ion binding' terms.

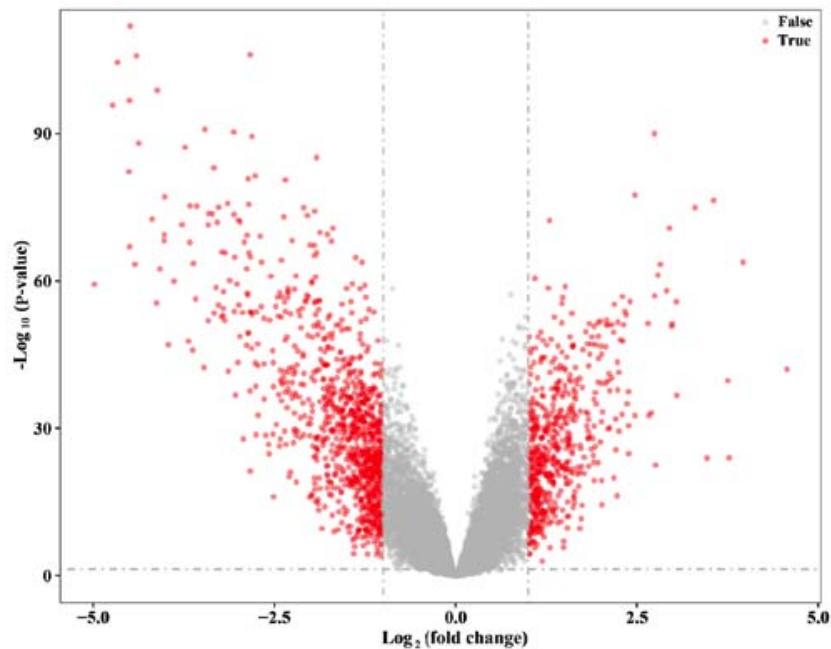

Figure 2. Volcano plot of DEGs A total of 1934 DEGs were identified with the thresholds of $\log _{2}$ fold change $>1$ or $<-1$ and adjusted P-value $<0.05$. All DEGs were marked as red dots. DEGs, differentially expressed genes. 
Table II. Top 50 upregulated differentially expressed genes.

\begin{tabular}{|c|c|c|c|c|c|c|c|}
\hline Probe ID & Gene & Fold change & $\begin{array}{l}\text { Adjusted } \\
\text { P-value }\end{array}$ & Probe ID & Gene & Fold change & $\begin{array}{l}\text { Adjusted } \\
\text { P-value }\end{array}$ \\
\hline 206239_s_at & SPINK1 & 4.567348387 & $9.90 \times 10^{-43}$ & 220491_at & HAMP & -4.983632921 & $4.89 \times 10^{-60}$ \\
\hline 211470_s_at & SULT1C2 & 3.963534914 & $1.47 \times 10^{-64}$ & 205866_at & FCN3 & -4.729403108 & $1.63 \times 10^{-96}$ \\
\hline 205815_at & REG3A & 3.770039412 & $1.10 \times 10^{-24}$ & 222484_s_at & CXCL14 & -4.664513026 & $2.76 \times 10^{-105}$ \\
\hline 209220_at & GPC3 & 3.752196862 & $1.99 \times 10^{-40}$ & 205984_at & CRHBP & -4.504769969 & $5.41 \times 10^{-83}$ \\
\hline 201291_s_at & TOP2A & 3.555152759 & $3.85 \times 10^{-77}$ & 207804_s_at & FCN2 & -4.498217224 & $1.69 \times 10^{-97}$ \\
\hline 206561_s_at & AKR1B10 & 3.468810343 & $1.21 \times 10^{-24}$ & 207201_s_at & SLC22A1 & -4.496482306 & $9.93 \times 10^{-68}$ \\
\hline 242881_x_at & DUXAP10 & 3.301086607 & $1.01 \times 10^{-75}$ & 220496_at & CLEC1B & -4.489579549 & $1.13 \times 10^{-112}$ \\
\hline 204602_at & DKK1 & 3.048004476 & $1.88 \times 10^{-37}$ & 217546_at & MT1M & -4.427062929 & $4.20 \times 10^{-64}$ \\
\hline 203820_s_at & IGF2BP3 & 3.043781559 & $1.76 \times 10^{-56}$ & 230478_at & OIT3 & -4.401727477 & $1.29 \times 10^{-106}$ \\
\hline 238021_s_at & CRNDE & 2.985078406 & $4.63 \times 10^{-52}$ & 1559573_at & LINC01093 & -4.372165201 & $7.87 \times 10^{-89}$ \\
\hline 209921_at & SLC7A11 & 2.980302339 & $1.66 \times 10^{-51}$ & 223699_at & CNDP1 & -4.185131569 & $2.30 \times 10^{-73}$ \\
\hline 213194_at & ROBO1 & 2.947155321 & $1.69 \times 10^{-71}$ & 229476_s_at & THRSP & -4.124366630 & $2.88 \times 10^{-56}$ \\
\hline 241418_at & NMRAL1P1 & 2.911349891 & $8.91 \times 10^{-59}$ & 1559065_a_at & CLEC4G & -4.114961753 & $1.36 \times 10^{-99}$ \\
\hline 223642_at & $\mathrm{ZIC} 2$ & 2.821525207 & $4.36 \times 10^{-64}$ & 206354_at & SLCO1B3 & -4.081557901 & $3.55 \times 10^{-63}$ \\
\hline 222608_s_at & ANLN & 2.787055993 & $6.53 \times 10^{-62}$ & 207102_at & AKR1D1 & -4.020716573 & $6.22 \times 10^{-69}$ \\
\hline 209875_s_at & SPP1 & 2.758625836 & $3.14 \times 10^{-23}$ & 1564706_s_at & GLS2 & -4.018224948 & $3.74 \times 10^{-70}$ \\
\hline 235004_at & RBM24 & 2.743050577 & $1.06 \times 10^{-57}$ & 207608_x_at & CYP1A2 & -4.013090089 & $7.43 \times 10^{-78}$ \\
\hline 212551_at & CAP2 & 2.739651435 & $9.30 \times 10^{-91}$ & 206727_at & C9 & -3.961247256 & $9.82 \times 10^{-48}$ \\
\hline 214612_x_at & MAGEA6 & 2.698311238 & $6.80 \times 10^{-34}$ & 209687_at & CXCL12 & -3.885309405 & $1.16 \times 10^{-60}$ \\
\hline 202422_s_at & ACSL4 & 2.663914238 & $2.38 \times 10^{-33}$ & 219014_at & PLAC8 & -3.774128748 & $3.42 \times 10^{-72}$ \\
\hline 219918_s_at & ASPM & 2.652302575 & $4.07 \times 10^{-52}$ & 207995_s_at & CLEC4M & -3.732362194 & $5.50 \times 10^{-88}$ \\
\hline 235763_at & SLC44A5 & 2.472658451 & $2.37 \times 10^{-33}$ & 231678_s_at & ADH4 & -3.682472263 & $1.77 \times 10^{-48}$ \\
\hline 207828_s_at & CENPF & 2.472581020 & $2.98 \times 10^{-78}$ & 205554_s_at & DNASE1L3 & -3.666231016 & $1.29 \times 10^{-68}$ \\
\hline 214710_s_at & CCNB1 & 2.403923370 & $1.64 \times 10^{-56}$ & 220801_s_at & HAO2 & -3.663835833 & $4.99 \times 10^{-76}$ \\
\hline 225681_at & CTHRC1 & 2.384346752 & $1.53 \times 10^{-25}$ & 211896_s_at & DCN & -3.625520940 & $1.19 \times 10^{-46}$ \\
\hline 212531_at & LCN2 & 2.383664937 & $3.04 \times 10^{-31}$ & 220432_s_at & CYP39A1 & -3.616388369 & $2.51 \times 10^{-64}$ \\
\hline 201890_at & RRM2 & 2.381259393 & $3.31 \times 10^{-38}$ & 220116_at & KCNN2 & -3.587327181 & $4.31 \times 10^{-57}$ \\
\hline 33323_r_at & SFN & 2.365919617 & $9.97 \times 10^{-36}$ & 205819_at & MARCO & -3.569710716 & $5.45 \times 10^{-76}$ \\
\hline 218009_s_at & PRC1 & 2.350952594 & $8.64 \times 10^{-54}$ & 202992_at & $\mathrm{C} 7$ & -3.473352727 & $3.89 \times 10^{-43}$ \\
\hline 204162_at & NDC80 & 2.332439517 & $1.54 \times 10^{-54}$ & 230135_at & HHIP & -3.458281369 & $1.29 \times 10^{-91}$ \\
\hline 219787_s_at & ECT2 & 2.313749179 & $8.57 \times 10^{-52}$ & 210328_at & GNMT & -3.412414732 & $1.25 \times 10^{-52}$ \\
\hline 207165_at & HMMR & 2.304640216 & $1.82 \times 10^{-48}$ & 213629_x_at & MT1F & -3.412271922 & $3.79 \times 10^{-72}$ \\
\hline 204825_at & MELK & 2.298883197 & $1.43 \times 10^{-57}$ & 214478_at & SPP2 & -3.405396399 & $1.74 \times 10^{-74}$ \\
\hline 203477_at & COL15A1 & 2.295323078 & $1.49 \times 10^{-33}$ & 205225_at & ESR1 & -3.355285624 & $2.09 \times 10^{-74}$ \\
\hline 203213_at & CDK1 & 2.271576100 & $9.42 \times 10^{-49}$ & 237350_at & TTC36 & -3.334397580 & $7.78 \times 10^{-84}$ \\
\hline 206626_x_at & SSX1 & 2.253448717 & $3.68 \times 10^{-30}$ & 214320_x_at & CYP2A6 & -3.332114585 & $3.55 \times 10^{-54}$ \\
\hline 207325_x_at & MAGEA1 & 2.252155461 & $3.97 \times 10^{-42}$ & 219954_s_at & GBA3 & -3.304920162 & $1.98 \times 10^{-59}$ \\
\hline 204720_s_at & DNAJC6 & 2.236082454 & $2.40 \times 10^{-52}$ & 207262_at & APOF & -3.287687923 & $1.09 \times 10^{-72}$ \\
\hline 204105_s_at & NRCAM & 2.232778933 & $1.57 \times 10^{-38}$ & 214621_at & GYS2 & -3.272101039 & $8.61 \times 10^{-56}$ \\
\hline 227892_at & PRKAA2 & 2.229124796 & $3.47 \times 10^{-44}$ & 206797_at & NAT2 & -3.270355860 & $8.59 \times 10^{-76}$ \\
\hline 227510_x_at & MALAT1 & 2.224576694 & $5.41 \times 10^{-17}$ & 242817_at & PGLYRP2 & -3.225627679 & $2.41 \times 10^{-53}$ \\
\hline 201468_s_at & NQO1 & 2.209490964 & $2.70 \times 10^{-26}$ & 205498_at & GHR & -3.223775643 & $1.20 \times 10^{-66}$ \\
\hline 205110_s_at & FGF13 & 2.207535230 & $1.23 \times 10^{-32}$ & 237390_at & ADRA1A & -3.213027372 & $8.85 \times 10^{-54}$ \\
\hline 223381_at & NUF2 & 2.186987390 & $5.66 \times 10^{-56}$ & 204704_s_at & ALDOB & -3.208187451 & $3.84 \times 10^{-55}$ \\
\hline 205476_at & CCL20 & 2.181812965 & $1.34 \times 10^{-20}$ & 209301_at & CA2 & -3.203130234 & $1.38 \times 10^{-58}$ \\
\hline 203755_at & BUB1B & 2.178984131 & $2.76 \times 10^{-50}$ & 206172_at & IL13RA2 & -3.185151095 & $1.11 \times 10^{-52}$ \\
\hline 218755_at & KIF20A & 2.153373571 & $8.72 \times 10^{-52}$ & 206210_s_at & CETP & -3.179352566 & $1.73 \times 10^{-66}$ \\
\hline 231265_at & COX7B2 & 2.150792051 & $5.69 \times 10^{-41}$ & 204428_s_at & LCAT & -3.145019681 & $1.47 \times 10^{-76}$ \\
\hline 221558_s_at & LEF1 & 2.122850025 & $2.29 \times 10^{-38}$ & 205363_at & BBOX1 & -3.135951206 & $2.16 \times 10^{-42}$ \\
\hline 225612_s_at & B3GNT5 & 2.117997087 & $7.16 \times 10^{-31}$ & 208147_s_at & CYP2C8 & -3.115222728 & $8.68 \times 10^{-61}$ \\
\hline
\end{tabular}

Table III. Top 50 downregulated differentially expressed genes. 
A

A

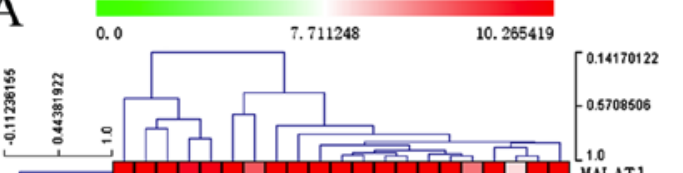

-

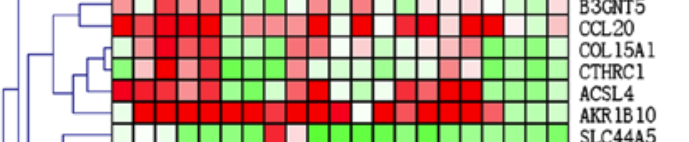
(2) \begin{tabular}{|c|c|c|c|c|c|c|c|c|c|}
\hline \\
\hline
\end{tabular}

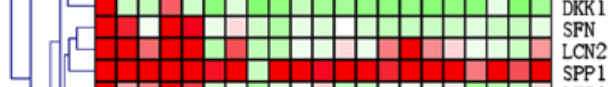

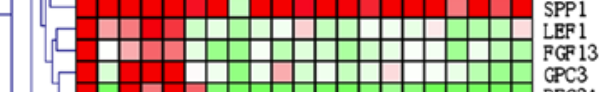
\begin{tabular}{|c|c|c|c|c|c|c|c|c|c|c|}
\hline \\
\hline
\end{tabular}

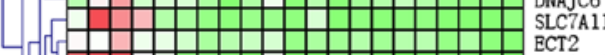
\begin{tabular}{|l|l|l|l|l|l|l|l|l|l|l} 
ECT2 \\
IGF 2BP3
\end{tabular}

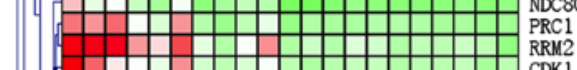

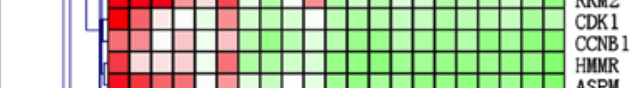

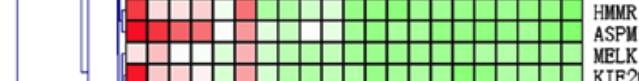
\begin{tabular}{|l|l|l|l|l|l|l|l|l|l|l|l}
\hline KELK \\
\hline
\end{tabular}

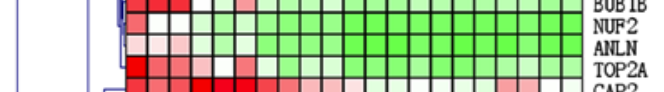
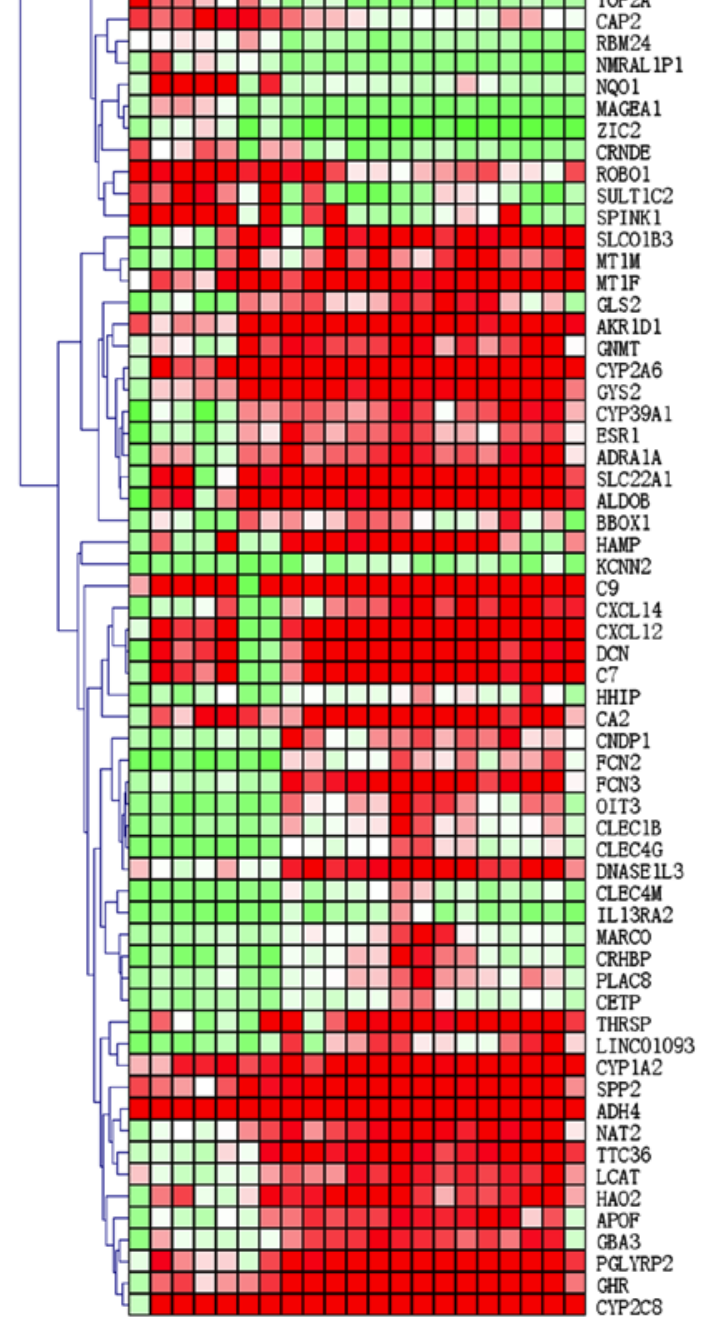

B
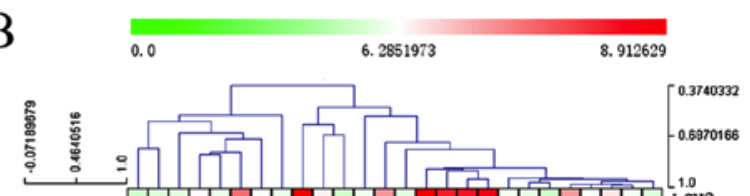
LCN2

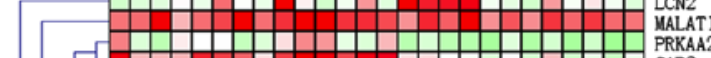
\begin{tabular}{|c|c|c|c|c|c|c|c|c|c|}
\hline & PRKAA2 \\
\hline & & & CAP2 & \\
\hline
\end{tabular}

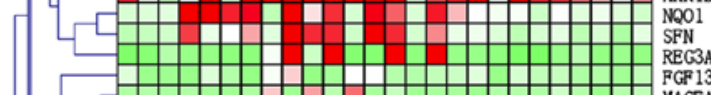

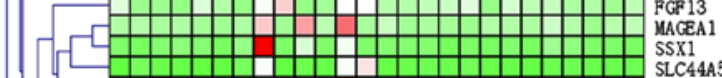

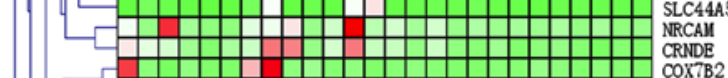
\begin{tabular}{|c|c|c|c|c|c|c|c|c|c|c|l}
\hline & \\
\hline
\end{tabular} (1) 4 (4)

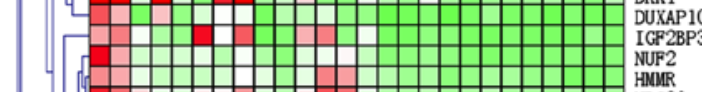

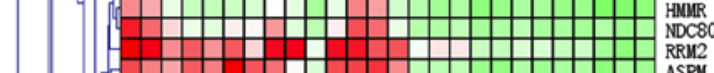

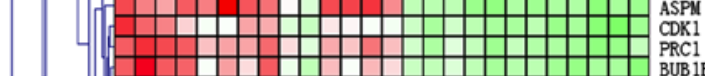

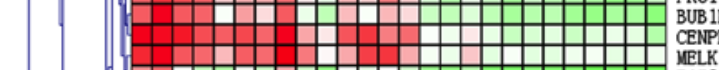

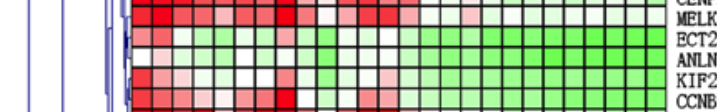

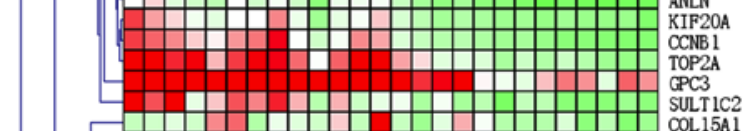

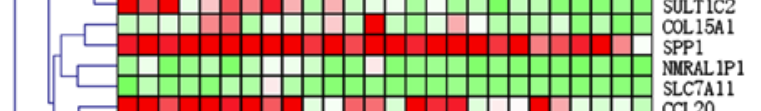

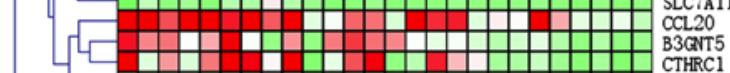

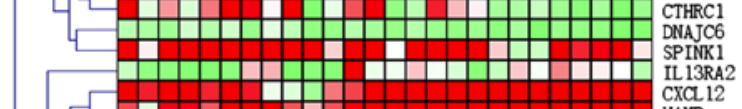

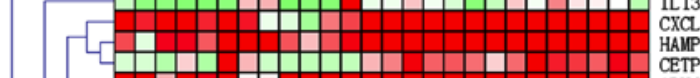
\begin{tabular}{|c|c|c|c|c|c|c|c|c|c|c|}
\hline \\
\hline
\end{tabular}

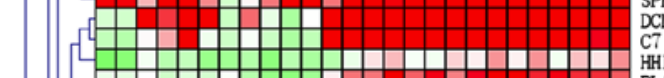
+010 H

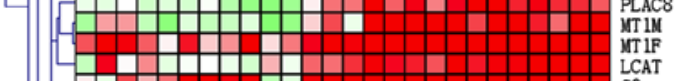

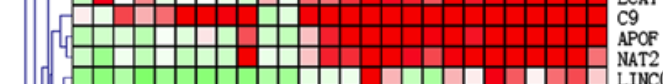

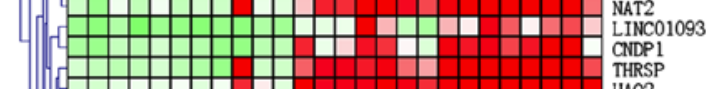

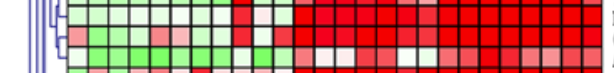

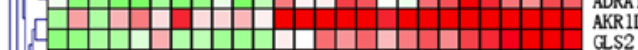

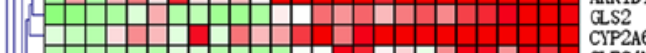
\begin{tabular}{|l|l|l|l|l|l|l|l|l|l|l|l}
\hline CLECAN \\
\hline
\end{tabular}

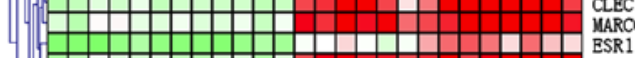

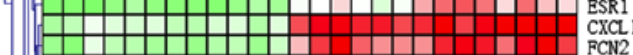

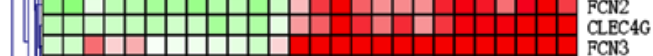

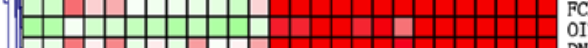

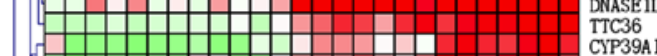

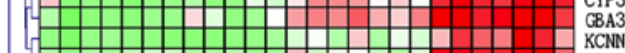

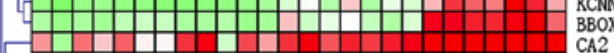

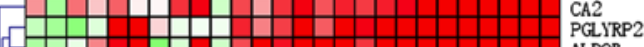

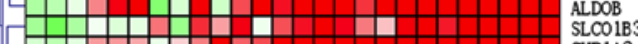

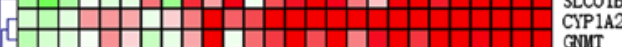

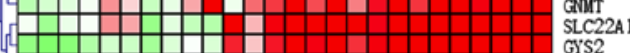

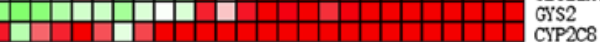

Figure 3. Heatmap of the expression profiles for the top 50 upregulated and downregulated DEGs in different subsets. The expression profiles for the top 50 upregulated and downregulated DEGs in (A) GSE17548 and (B) GSE84402. DEGs, differentially expressed genes. The colours represent the expression level of the genes, and the higher the expression level, the darker the colour: Red, upregulated; green, downregulated. 

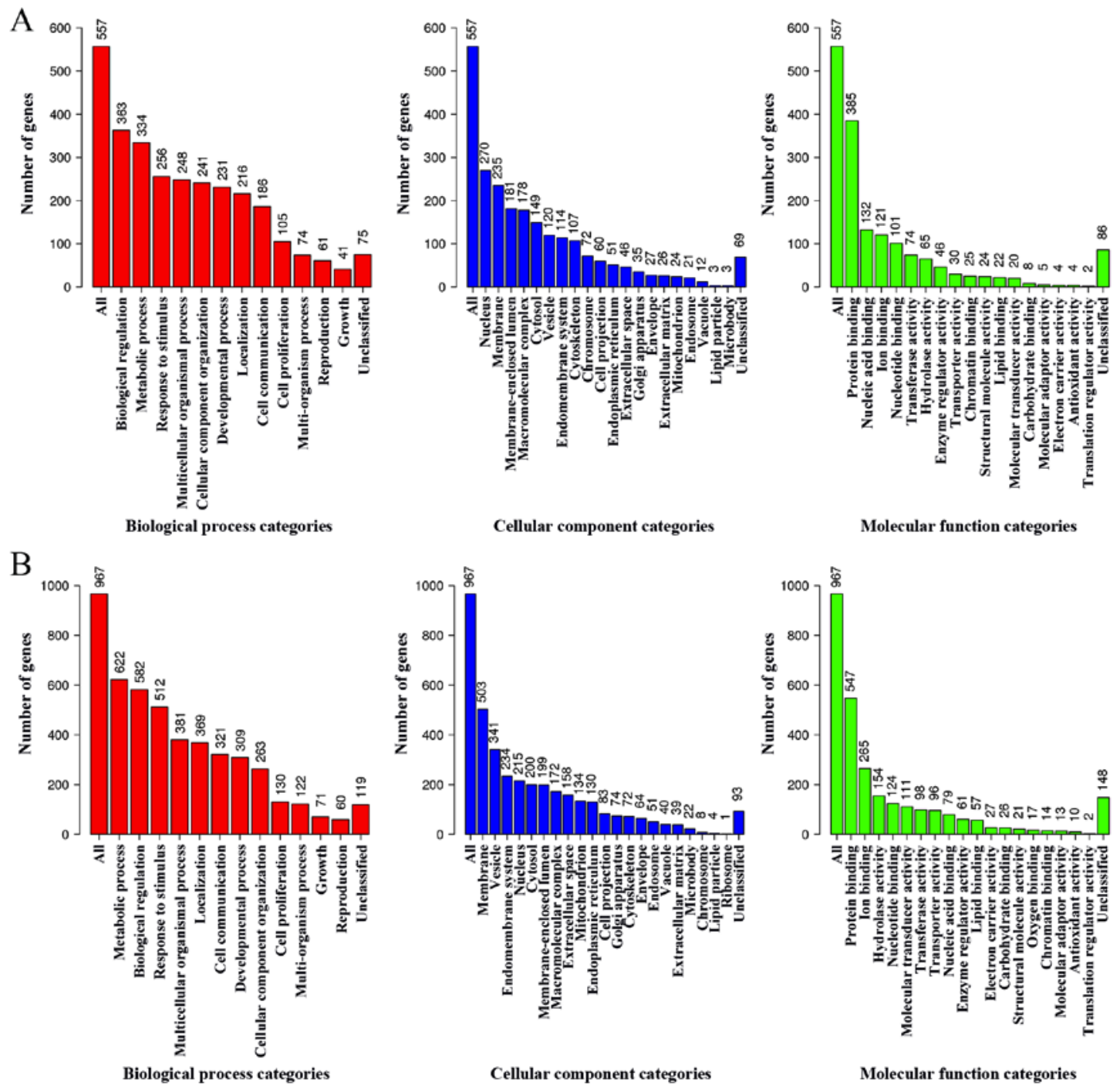

Figure 4. GO terms of the DEGs. Each Biological Process, Cellular Component and Molecular Function category is represented by red, blue and green bars, respectively. The height of the bar represents the number of DEGs observed in the category. The GO terms of (A) upregulated genes and (B) downregulated genes. DEGs, differentially expressed genes; GO, Gene Ontology.

Table IV. Top 10 enriched KEGG pathway terms of upregulated differentially expressed genes.

\begin{tabular}{llcc}
\hline KEGG ID & \multicolumn{1}{c}{ KEGG pathway } & $\begin{array}{c}\text { No. ofss } \\
\text { genes }\end{array}$ & P-value \\
\hline hsa04110 & Cell cycle & 24 & $2.51 \times 10^{-14}$ \\
hsa05222 & Small cell lung cancer & 13 & $5.70 \times 10^{-07}$ \\
hsa05200 & Pathways in cancer & 28 & $4.02 \times 10^{-06}$ \\
hsa04512 & ECM-receptor interaction & 11 & $1.42 \times 10^{-05}$ \\
hsa04115 & p53 signaling pathway & 10 & $1.76 \times 10^{-05}$ \\
hsa04151 & PI3K-Akt signaling & 21 & $4.69 \times 10^{-04}$ \\
& pathwayss & & \\
hsa04510 & Focal adhesion & 15 & $4.89 \times 10^{-04}$ \\
hsa05146 & Amoebiasis & 9 & $1.74 \times 10^{-03}$ \\
hsa05213 & Endometrial cancer & 6 & $2.97 \times 10^{-03}$ \\
hsa05218 & Melanoma & 7 & $3.37 \times 10^{-03}$ \\
& &
\end{tabular}

KEGG, Kyoto Encyclopedia of Genes and Genomes; ECM, extracellular matrix.
In addition, the top 10 enriched KEGG pathway terms of the up- and downregulated genes are provided in Tables IV and V, respectively. The upregulated DEGs were primarily associated with the 'cell cycle', whereas the downregulated DEGs were primarily associated with the 'metabolic pathways'.

Notably, there were six genes with $\log _{2}$-fold change $>2$ in DEGs enriched in the 'cell cycle' pathway: $S F N, B U B 1 B$, $T T K, C C N B 1, C D K 1$ and $C D C 20$. Differential expression analysis was performed on the aforementioned six genes in non-HBV tissues from two independent HCC datasets (TCGA and GSE76427) on different platforms (TCGA, Illumina RNA Sequencing; GSE76427, Illumina HumanHT-12 V4.0 expression beadchip). None of these genes had a $\log _{2}$-fold change $>2$ (Table VI), which demonstrates that the high expression of these six DEGs in HBV-associated HCC is more significant compared with non-HBV HCC.

PPI network analysis of the DEGs. To further understand the biological meaning of the DEGs identified by the top KEGG pathways at the protein level, two PPI networks for the proteins encoded by the DEGs in the top pathways were constructed. 
Table V. Top 10 enriched KEGG pathway terms of downregulated differentially expressed genes.

\begin{tabular}{llrr}
\hline KEGG ID & \multicolumn{1}{c}{ KEGG pathway } & No. of genes & P-value \\
\hline hsa01100 & Metabolic pathways & 185 & $<0.01$ \\
hsa04610 & Complement and coagulation cascades & 33 & $<0.01$ \\
hsa05204 & Chemical carcinogenesis & 27 & $5.49 \times 10^{-12}$ \\
hsa01200 & Carbon metabolism & 30 & $2.04 \times 10^{-10}$ \\
hsa00260 & Glycine, serine and threonine metabolism & 17 & $5.18 \times 10^{-10}$ \\
hsa00380 & Tryptophan metabolism & 17 & $5.18 \times 10^{-10}$ \\
hsa05150 & Staphylococcus aureus infection & 20 & $6.30 \times 10^{-10}$ \\
hsa00830 & Retinol metabolism & 21 & $1.95 \times 10^{-09}$ \\
hsa00071 & Fatty acid degradation & 17 & $3.05 \times 10^{-09}$ \\
hsa00250 & Alanine, aspartate and glutamate metabolism & 15 & $4.8 \times 10^{-09}$
\end{tabular}

KEGG, Kyoto Encyclopedia of Genes and Genomes.

Table VI. Differential expression of six differentially expressed genes in different datasets.

\begin{tabular}{|c|c|c|c|c|c|c|}
\hline \multirow[b]{2}{*}{ Gene } & \multicolumn{2}{|c|}{ Large HBV cohort } & \multicolumn{2}{|c|}{ GSE76427 } & \multicolumn{2}{|c|}{ TCGA } \\
\hline & Fold change & Adj. P-value & Fold change & Adj. P-value & Fold change & Adj. P-value \\
\hline CCNB 1 & 2.403923 & $1.64 \times 10^{-56}$ & 0.587062 & $1.36 \times 10^{-11}$ & 0.607121 & $1.92 \times 10^{-11}$ \\
\hline SFN & 2.365920 & $9.97 \times 10^{-36}$ & 0.880437 & $3.36 \times 10^{-03}$ & 0.676444 & $2.14 \times 10^{-05}$ \\
\hline CDK1 & 2.271576 & $9.42 \times 10^{-49}$ & 0.802341 & $5.35 \times 10^{-11}$ & 0.769405 & $1.93 \times 10^{-11}$ \\
\hline BUB1B & 2.178984 & $2.76 \times 10^{-50}$ & 1.121998 & $5.27 \times 10^{-12}$ & 0.234839 & $9.11 \times 10^{-07}$ \\
\hline TTK & 2.066772 & $2.45 \times 10^{-47}$ & 1.587680 & $3.34 \times 10^{-13}$ & 0.791737 & $2.17 \times 10^{-12}$ \\
\hline CDC20 & 2.012448 & $7.80 \times 10^{-46}$ & 1.073711 & $8.29 \times 10^{-09}$ & 1.712167 & $4.21 \times 10^{-10}$ \\
\hline
\end{tabular}

HBV, hepatitis B virus; Adj., adjusted; TCGA, The Cancer Genome Atlas.

The PPI network of the 'cell cycle' consisted of 24 nodes and 85 edges, whereas the PPI network of the 'metabolic pathways' consisted of 184 nodes and 566 edges (Fig. 5).

\section{Discussion}

The present study specifically focused on HBV-infected patients, which is different from the previous studies on HCC regardless of etiology $(19,27)$. A total of 682 upregulated DEGs and 1,252 downregulated DEGs were identified in HCC tissues compared with nontumor hepatic tissues in $321 \mathrm{HBV}$-positive samples. KEGG analyses demonstrated that the upregulated DEGs were enriched in signaling pathways such as the cell cycle, p53 signaling pathway and extracellular matrix-receptor interaction. A previous study showed that HBV infection deregulates the cell cycle pathway (28). Notably, there were 6 genes with a $\log _{2}$-fold change $>2$ among the DEGs enriched in the 'cell cycle' pathway: $S F N, B U B 1 B, T T K, C C N B 1, C D K 1$ and $C D C 20$.

SFN (14-3-3б) protein is a member of the 14-3-3 superfamily (29). SFN has been found to play a key role in various vital regulatory processes, such as cell cycle regulation and signaling pathways (30). In a previous study, high expression of SFN was detected in HCC tissues but not in adjacent nontumor tissues, which indicated an association between SFN and HCC (31). In another study, SFN exhibited high diagnostic accuracy in the differentiation of $\mathrm{HCC}$ from nontumorous hepatocytes (32).

BUB1B (encoding BUBR1) is an important component in the SAC protein family, which has been found to be involved in several forms of human cancer, such as lung cancer and breast cancer $(33,34)$. However, the contradiction of BUB1B expression in cancer cells remains controversial. Low expression of BUB1B is associated with the poor survival of patients with colon adenocarcinomas and lung cancer, however overexpression of BUB1B contributes to the progression and recurrence of gastric cancer and bladder cancer (35). However, several studies showed that the overexpression of BUB1B is associated with worse prognosis in patients with $\operatorname{HCC}(36,37)$.

TTK, a dual-specific protein kinase participating in the $\mathrm{p} 53$ pathway, has been found to be involved in several cancer types by modulating the mitotic checkpoint (38). A previous study by Miao et al (39), regarding HBV-associated HCC, reported TTK as a promising prognostic marker of HCC. TTK alone can accurately predict the recurrence rate and recurrence time. These findings on TTK drew interest and resulted in further studies on cancer (40-42). The results of the present study supported the conclusion of the study by Miao et al (39). 
A

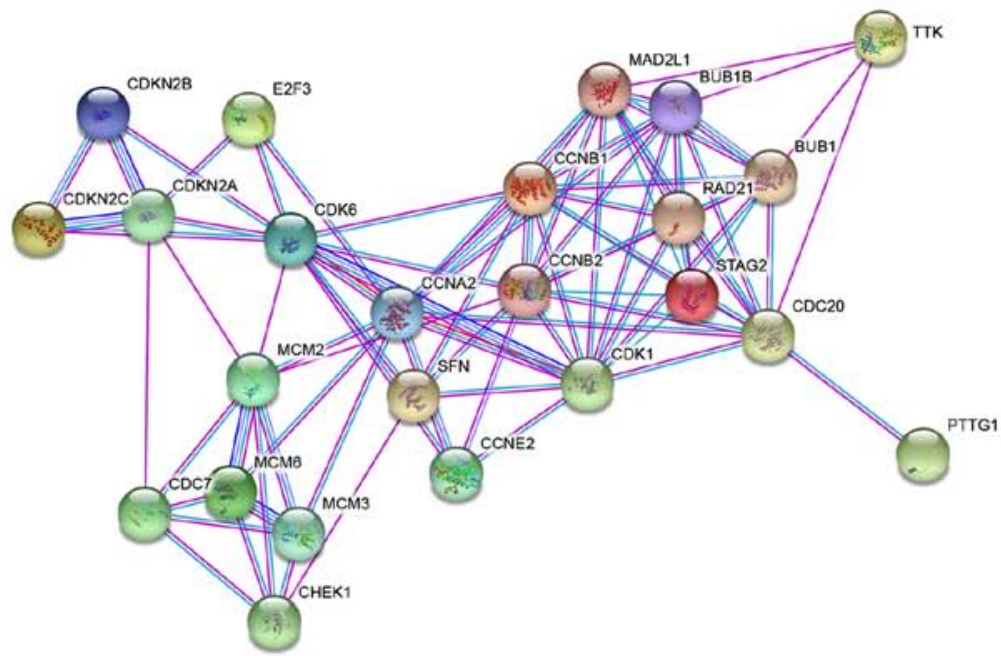

$\mathrm{B}$

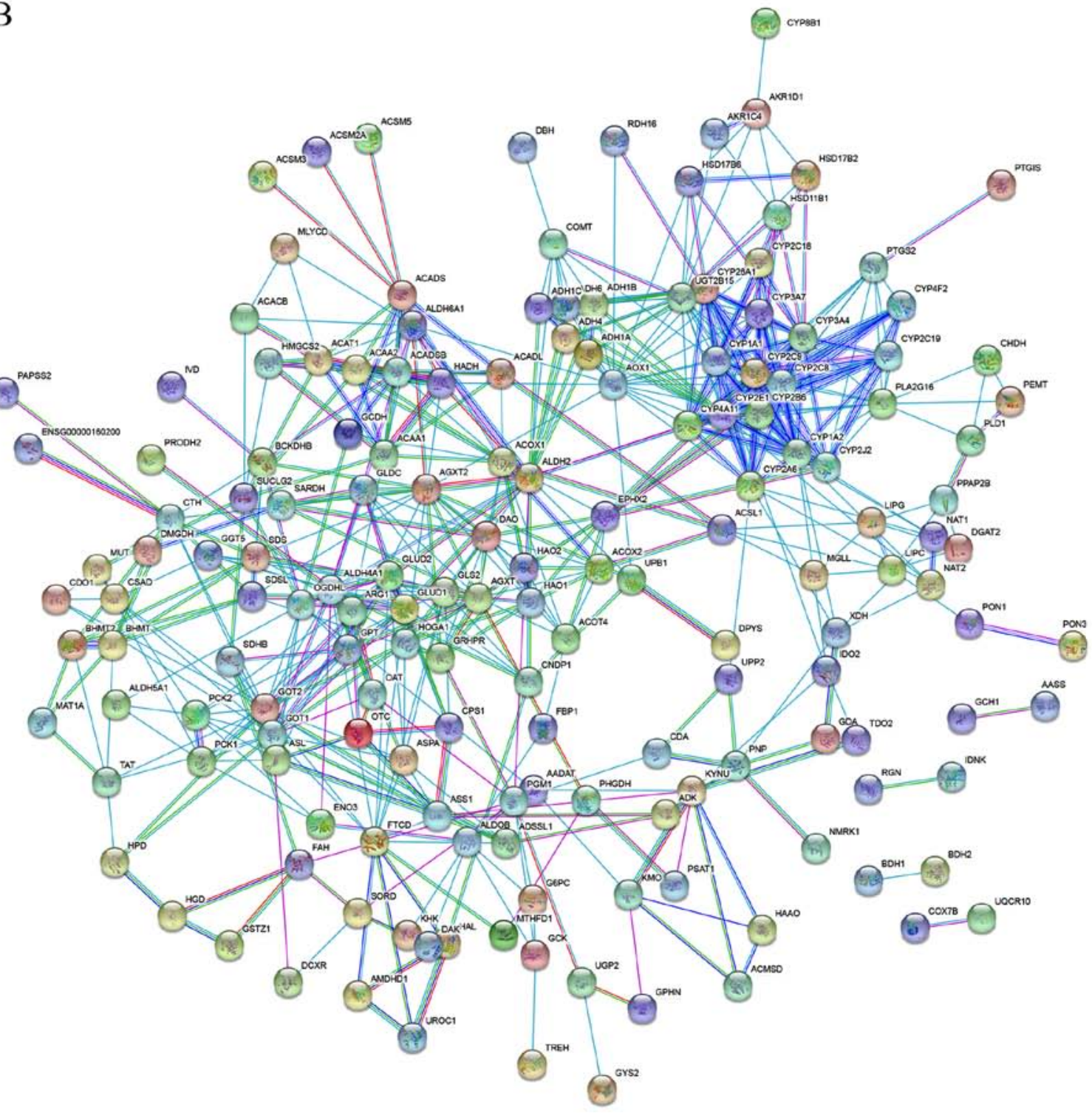

Figure 5. PPI networks of the DEGs. PPI network of DEGs in (A) 'Cell cycle' and (B) in 'Metabolic pathways'. PPI, protein-protein interaction; DEGs, differentially expressed genes.

CCNB1 plays an integral role in regulating the $\mathrm{G}_{2} / \mathrm{M}$ transition in the cell cycle (43). Several studies have found an elevated expression of CCNB1 in different cancer types, such as breast cancer (44), non-small cell carcinoma (45) and gastric cancer (46). In a previous study, CCNB1 was reported as an independent risk factor of recurrence in patients with HBV-associated HCC 
following surgery (47). However, it is still unclear how CCNB1 contributes to oncogenesis and tumor progression.

CDK1 is required for the role of CCNB1 in the $\mathrm{G}_{2} / \mathrm{M}$ transition and mitosis resumption (48). Cheng et al (49) conducted in vitro experiments, which demonstrated that HBV could activate the CCNB1-CDK1 kinase in HCC cells. In other studies, CCNB1 and CDK1 were found to be upregulated in the HCC tissues of HBV-positive patients (50). Moreover, overexpression of these two genes is associated with poor prognosis. CDK1 was considered important as CCNB1, since it could affect both overall survival and recurrence-free survival of HBV-positive patients with HCC (51).

CDC20 functions as a regulatory protein that interacts with several other proteins at multiple points in the cell cycle. Chae et al (52) demonstrated that HBV-infection could attenuate the association between BubR1 and CDC20, thus preventing CDC20 from performing its original function, which provided a novel view on the development of HBV-associated HCC.

The high expression of the six DEGs was more significant in HBV-associated HCC than in non-HBV HCC, and was validated in two independent $\mathrm{HCC}$ datasets. In future studies, clinical HCC samples should be collected in order to verify that these genes are affected by HBV infection.

The downregulated DEGs were enriched in signaling pathways such as 'carbon metabolism', 'glycine, serine and threonine metabolism', 'tryptophan metabolism', 'retinol metabolism' and 'alanine, aspartate and glutamate metabolism'. A previous study reported that HBV-infection could induce alterations in metabolic signaling pathways. The consequences may alter normal hepatocyte metabolism, thus contributing to the progression of $\mathrm{HBV}$-associated carcinogenesis (53).

In conclusion, the present study identified several DEGs in HCC tissues compared with nontumor tissues from HBV-infected patients, based on a large cohort. Based on the DEGs, several key pathways were identified. The interactions of the DEGs in the pathways were also presented by PPI networks. Some results were consistent with previous studies $(39,50)$. Furthermore, the present study provides new insights into the specific etiology of HCC and molecular mechanisms for the transformation of nontumor hepatic tissues into HCC tissues, in patients with a history of HBV infection. Importantly, these results may provide several potential therapeutic targets for targeted therapy in these patients, which could aid early diagnosis and treatment of HCC.

\section{Acknowledgements}

Not applicable.

\section{Funding}

This study was supported in part by grants from the Natural Science Foundation of Jiangxi Province (grant no. 20181BBG78042).

\section{Availability of data and materials}

The datasets used and/or analyzed during the present study are available from the corresponding author on reasonable request.

\section{Authors' contributions}

XZ, LW and YY conceived and designed the study. LW analyzed the data. XZ contributed to literature review. $\mathrm{XZ}$ and LW wrote the manuscript. XZ and YY reviewed and edited the manuscript. All authors read and approved the final manuscript.

\section{Ethics approval and consent to participate}

Not applicable.

\section{Patient consent for publication}

Not applicable.

\section{Competing interests}

The authors declare that they have no competing interests.

\section{References}

1. Wu CC, Wu DW, Lin YY, Lin PL and Lee H: Hepatitis B virus X protein represses LKB1 expression to promote tumor progression and poor postoperative outcome in hepatocellular carcinoma. Surgery 163: 1040-1046, 2018.

2. Duan L, Wu R, Zhang X, Wang D, You Y, Zhang Y, Zhou L and Chen W: HBx-induced S100A9 in NF- $\kappa$ B dependent manner promotes growth and metastasis of hepatocellular carcinoma cells. Cell Death Dis 9: 629, 2018.

3. Tsunematsu S, Suda G, Yamasaki K, Kimura M, Izumi T, Umemura M, Ito J, Sato F, Nakai M, Sho T, et al: Hepatitis B virus $X$ protein impairs alpha-interferon signaling via up-regulation of suppressor of cytokine signaling 3 and protein phosphatase 2A. J Med Virol 89: 267-275, 2017.

4. Kapoor NR, Chadha R, Kumar S, Choedon T, Reddy VS and Kumar V: The HBx gene of hepatitis B virus can influence hepatic microenvironment via exosomes by transferring its mRNA and protein. Virus Res 240: 166-174, 2017.

5. Casciano JC and Bouchard MJ: Hepatitis B virus X protein modulates cytosolic $\mathrm{Ca}(2+)$ signaling in primary human hepatocytes. Virus Res 246: 23-27, 2018.

6. Huang XY, Li D, Chen ZX, Huang YH, Gao WY, Zheng BY and Wang XZ: Hepatitis B Virus X protein elevates Parkin-mediated mitophagy through Lon Peptidase in starvation. Exp Cell Res 368: 75-83, 2018.

7. Hensel KO, Cantner F, Bangert F, Wirth S and Postberg J: Episomal HBV persistence within transcribed host nuclear chromatin compartments involves HBx. Epigenetics Chromatin 11: 34, 2018.

8. Li C, Lin C, Cong X and Jiang Y: PDK1-WNK1 signaling is affected by HBx and involved in the viability and metastasis of hepatic cells. Oncol Lett 15: 5940-5946, 2018.

9. Jin Y, Wu D, Yang W, Weng M, Li Y, Wang X, Zhang X, Jin X and Wang T: Hepatitis B virus x protein induces epithelial-mesenchymal transition of hepatocellular carcinoma cells by regulating long non-coding RNA. Virol J 14: 238, 2017.

10. Xu F, Song H, Xiao Q, Li N, Zhang H, Cheng G and Tan G: Type III interferon-induced CBFbeta inhibits HBV replication by hijacking HBx. Cell Mol Immunol 17: 357-366, 2019.

11. Liu Y, Yao W, Si L, Hou J, Wang J, Xu Z, Li W, Chen J, Li R, Li P, et al: Chinese herbal extract Su-duxing had potent inhibitory effects on both wild-type and entecavir-resistant hepatitis B virus (HBV) in vitro and effectively suppressed HBV replication in mouse model. Antiviral Res 155: 39-47, 2018.

12. Ko C, Chakraborty A, Chou WM, Hasreiter J, Wettengel JM, Stadler D, Bester R, Asen T, Zhang K, Wisskirchen K, et al: Hepatitis $B$ virus genome recycling and de novo secondary infection events maintain stable cccDNA levels. J Hepatol 69: 1231-1241, 2018.

13. Han Q, Wang X, Liao X, Han C, Yu T, Yang C, Li G, Han B, Huang K, Zhu G, et al: Diagnostic and prognostic value of WNT family gene expression in hepatitis B virusrelated hepatocellular carcinoma. Oncol Rep 42: 895-910, 2019. 
14. Tian Y and $\mathrm{Ou} \mathrm{JH}$ : Genetic and epigenetic alterations in hepatitis B virus-associated hepatocellular carcinoma. Virol Sin 30: 85-91, 2015.

15. Xie X, Xu X, Sun C and Yu Z: Hepatitis B virus X protein promotes proliferation of hepatocellular carcinoma cells by upregulating miR-181b by targeting ING5. Biol Chem 399: 611-619, 2018.

16. Hamamoto H, Maemura K, Matsuo K, Taniguchi K, Tanaka Y, Futaki S, Takeshita A, Asai A, Hayashi M, Hirose Y, et al: Delta-like 3 is silenced by HBx via histone acetylation in HBV-associated HCCs. Sci Rep 8: 4842, 2018.

17. Tang Q, Wang Q, Zhang Q, Lin SY, Zhu Y, Yang X and Guo AY: Gene expression, regulation of DEN and HBx induced HCC mice models and comparisons of tumor, para-tumor and normal tissues. BMC Cancer 17: 862, 2017.

18. He X, Zhang C, Shi C and Lu Q: Meta-analysis of mRNA expression profiles to identify differentially expressed genes in lung adenocarcinoma tissue from smokers and non-smokers. Oncol Rep 39: 929-938, 2018.

19. Yildiz G, Arslan-Ergul A, Bagislar S, Konu O, Yuzugullu H, Gursoy-Yuzugullu O, Ozturk N, Ozen C, Ozdag H, Erdal E, et al: Genome-wide transcriptional reorganization associated with senescence-to-immortality switch during human hepatocellular carcinogenesis. PLoS One 8: e64016, 2013.

20. Melis M, Diaz G, Kleiner DE, Zamboni F, Kabat J, Lai J, Mogavero G, Tice A, Engle RE, Becker S, et al: Viral expression and molecular profiling in liver tissue versus microdissected hepatocytes in hepatitis B virus-associated hepatocellular carcinoma. J Transl Med 12: 230, 2014

21. Schulze K, Imbeaud S, Letouze E, Alexandrov LB, Calderaro J, Rebouissou S, Couchy G, Meiller C, Shinde J, Soysouvanh F, et al: Exome sequencing of hepatocellular carcinomas identifies new mutational signatures and potential therapeutic targets. Nat Genet 47: 505-511, 2015.

22. Wang M, Gong Q, Zhang J, Chen L, Zhang Z, Lu L, Yu D, Han Y, Zhang D, Chen $\mathrm{P}$, et al: Characterization of gene expression profiles in HBV-related liver fibrosis patients and identification of ITGBL1 as a key regulator of fibrogenesis. Sci Rep 7: 43446, 2017.

23. Wang H, Huo X, Yang XR, He J, Cheng L, Wang N, Deng X, Jin $\mathrm{H}$, Wang $\mathrm{N}$, Wang $\mathrm{C}$, et al: STAT3-mediated upregulation of IncRNA HOXD-AS1 as a ceRNA facilitates liver cancer metastasis by regulating SOX4. Mol Cancer 16: 136, 2017.

24. Irizarry RA, Hobbs B, Collin F, Beazer-Barclay YD, Antonellis KJ, Scherf U and Speed TP: Exploration, normalization, and summaries of high density oligonucleotide array probe level data. Biostatistics 4: 249-264, 2003.

25. Grinchuk OV, Yenamandra SP, Iyer R, Singh M, Lee HK, Lim KH, Chow PK and Kuznetsov VA: Tumor-adjacent tissue co-expression profile analysis reveals pro-oncogenic ribosomal gene signature for prognosis of resectable hepatocellular carcinoma. Mol Oncol 12: 89-113, 2018.

26. Phipson B, Lee S, Majewski IJ, Alexander WS and Smyth GK: Robust hyperparameter estimation protects against hypervariable genes and improves power to detect differential expression. Ann Appl Stat 10: 946-963, 2016.

27. He B, Yin J, Gong S, Gu J, Xiao J, Shi W, Ding W and He Y: Bioinformatics analysis of key genes and pathways for hepatocellular carcinoma transformed from cirrhosis. Medicine (Baltimore) 96: e6938, 2017.

28. Xia Y, Cheng X, Li Y, Valdez K, Chen W and Liang TJ: Hepatitis B Virus Deregulates the Cell Cycle To Promote Viral Replication and a Premalignant Phenotype. J Virol 92, 2018.

29. Chen XL, Zhou L, Yang J, Shen FK, Zhao SP and Wang YL: Hepatocellular carcinoma-associated protein markers investigated by MALDI-TOF MS. Mol Med Rep 3: 589-596, 2010.

30. Lewis AG, Flanagan J, Marsh A, Pupo GM, Mann G, Spurdle AB, Lindeman GJ, Visvader JE, Brown MA and Chenevix-Trench G; Kathleen Cuningham Foundation Consortium for Research into Familial Breast Cancer: Mutation analysis of FANCD2, BRIP1/BACH1, LMO4 and SFN in familial breast cancer. Breast Cancer Res 7: R1005-1016, 2005.

31. Lee IN, Chen CH, Sheu JC, Lee HS, Huang GT, Yu CY, $\mathrm{Lu}$ FJ and Chow LP: Identification of human hepatocellular carcinoma-related biomarkers by two-dimensional difference gel electrophoresis and mass spectrometry. J Proteome Res 4: 2062-2069, 2005.

32. Reis H, Putter C, Megger DA, Bracht T, Weber F, Hoffmann AC, Bertram S, Wohlschlager J, Hagemann S, Eisenacher M, et al: A structured proteomic approach identifies 14-3-3 sigma as a novel and reliable protein biomarker in panel based differential diagnostics of liver tumors. Biochim Biophys Acta 1854: 641-650, 2015.
33. Saeki A, Tamura S, Ito N, Kiso S, Matsuda Y, Yabuuchi I, Kawata S and Matsuzawa Y: Frequent impairment of the spindle assembly checkpoint in hepatocellular carcinoma. Cancer 94: 2047-2054, 2002.

34. Seike M, Gemma A, Hosoya Y, Hosomi Y, Okano T, Kurimoto F, Uematsu K, Takenaka K, Yoshimura A, Shibuya M, et al: The promoter region of the human BUBR1 gene and its expression analysis in lung cancer. Lung Cancer 38: 229-234, 2002.

35. Zhuang L, Yang Z and Meng Z: Upregulation of BUB1B CCNB1, CDC7, CDC20, and MCM3 in tumor tissues predicted worse overall survival and disease-free survival in hepatocellular carcinoma patients. Biomed Res Int 2018: 7897346, 2018.

36. Liu AW, Cai J, Zhao XL, Xu AM, Fu HQ, Nian H and Zhang SH: The clinicopathological significance of BUBR1 overexpression in hepatocellular carcinoma. J Clin Pathol 62: 1003-1008, 2009.

37. Sun B, Lin G, Ji D, Li S, Chi G and Jin X: Dysfunction of sister chromatids separation promotes progression of hepatocellular carcinoma according to analysis of gene expression profiling. Front Physiol 9: 1019, 2018.

38. Janssen A, van der Burg M, Szuhai K, Kops GJ and Medema RH: Chromosome segregation errors as a cause of DNA damage and structural chromosome aberrations. Science 333: 1895-1898, 2011.

39. Miao R, Luo H, Zhou H, Li G, Bu D, Yang X, Zhao X, Zhang H, Liu S, Zhong Y, et al: Identification of prognostic biomarkers in hepatitis B virus-related hepatocellular carcinoma and stratification by integrative multi-omics analysis. J Hepatol 61: 840-849, 2014

40. Pineda-Solis K and McAlister V: Wading through the noise of 'multi-omics' to identify prognostic biomarkers in hepatocellular carcinoma. Hepatobiliary Surg Nutr 4: 293-294, 2015.

41. Baffy G: Decoding multifocal hepatocellular carcinoma: An opportune pursuit. Hepatobiliary Surg Nutr 4: 206-210, 2015.

42. Feo F and Pascale RM: Multifocal hepatocellular carcinoma: Intrahepatic metastasis or multicentric carcinogenesis? Ann Transl Med 3: 4, 2015.

43. Wang G, Chen H, Huang M, Wang N, Zhang J, Zhang Y, Bai G, Fong WF, Yang $\mathrm{M}$ and Yao $\mathrm{X}$ : Methyl protodioscin induces G2/M cell cycle arrest and apoptosis in HepG2 liver cancer cells. Cancer Lett 241: 102-109, 2006.

44. Nimeus-Malmstrom E, Koliadi A, Ahlin C, Holmqvist M, Holmberg L, Amini RM, Jirstrom K, Warnberg F, Blomqvist C, Ferno M and Fjällskog ML: Cyclin B1 is a prognostic proliferation marker with a high reproducibility in a population-based lymph node negative breast cancer cohort. Int J Cancer 127: 961-967, 2010.

45. Soria JC, Jang SJ, Khuri FR, Hassan K, Liu D, Hong WK and Mao L: Overexpression of cyclin B1 in early-stage non-small cell lung cancer and its clinical implication. Cancer Res 60: 4000-4004, 2000.

46. Begnami MD, Fregnani JH, Nonogaki S and Soares FA: Evaluation of cell cycle protein expression in gastric cancer: cyclin B1 expression and its prognostic implication. Hum Pathol 41: 1120-1127, 2010.

47. Weng L, Du J, Zhou Q, Cheng B, Li J, Zhang D and Ling C: Identification of cyclin B1 and Sec62 as biomarkers for recurrence in patients with $\mathrm{HBV}$-related hepatocellular carcinoma after surgical resection. Mol Cancer 11: 39, 2012.

48. Fang Y, Yu H, Liang X, Xu J and Cai X: Chk1-induced CCNB1 overexpression promotes cell proliferation and tumor growth in human colorectal cancer. Cancer Biol Ther 15: 1268-1279, 2014.

49. Cheng P, Li Y, Yang L, Wen Y, Shi W, Mao Y, Chen P, Lv H, Tang $\mathrm{Q}$ and Wei $\mathrm{Y}$ : Hepatitis $\mathrm{B}$ virus $\mathrm{X}$ protein $(\mathrm{HBx})$ induces $\mathrm{G} 2 / \mathrm{M}$ arrest and apoptosis through sustained activation of cyclin B1-CDK1 kinase. Oncol Rep 22: 1101-1107, 2009.

50. Chen QF, Xia JG, Li W, Shen LJ, Huang T and Wu P: Examining the key genes and pathways in hepatocellular carcinoma development from hepatitis B viruspositive cirrhosis. Mol Med Rep 18: 4940-4950, 2018.

51. Li H, Zhao X, Li C, Sheng C and Bai Z: Integrated analysis of lncRNA-associated ceRNA network reveals potential biomarkers for the prognosis of hepatitis B virus-related hepatocellular carcinoma. Cancer Manag Res 11: 877-897, 2019.

52. Chae S, Ji JH, Kwon SH, Lee HS, Lim JM, Kang D, Lee CW and Cho H: HBxAPalpha/Rsf-1-mediated HBx-hBubR1 interactions regulate the mitotic spindle checkpoint and chromosome instability. Carcinogenesis 34: 1680-1688, 2013.

53. Slagle BL and Bouchard MJ: Role of HBx in hepatitis B virus persistence and its therapeutic implications. Curr Opin Virol 30: 32-38, 2018.

This work is licensed under a Creative Commons Attribution-NonCommercial-NoDerivatives 4.0 International (CC BY-NC-ND 4.0) License. 